\title{
The prevalence of multiple sclerosis in the Leeds Health Authority
}

\author{
H L Ford, E Gerry, C M Airey, A Vail, M H Johnson, D R R Williams
}

\begin{abstract}
Objectives-To determine the prevalence of multiple sclerosis in the Leeds Health District.

Methods-Multiple sources of case ascertainment were used-namely, neurology departments, hospital episode statistics, general practitioners, the Leeds branch of the Multiple Sclerosis Society, the West Yorkshire Multiple Sclerosis Therapy Centre, community physiotherapists and occupational therapists, the Leeds Wheelchair Centre, and the Young Disabled Unit. Data collection was from retrospective analysis of hospital and primary care case records. A population based incidence register was established by prospectively registering all new patients with diagnoses of multiple sclerosis.

Results-On prevalence day, 30 April 1996, 712 people with multiple sclerosis were identified living in Leeds (population 732061 ), giving a prevalence of $97 / 10^{5}$. The prevalence for definite and probable multiple sclerosis was $84 / 10^{5}$, and for suspected multiple sclerosis it was $13 / 10^{5}$. The sex ratio of prevalent people with multiple sclerosis was 2.79 to 1 women to men. The mean age of prevalent cases was 51 years, the mean age at symptom onset was 34 years, and the mean duration of disease was 16 years. Forty cases were prospectively reported as incident cases from 1 November 1995 to 1 February 1996.

Conclusions-The prevalence of multiple sclerosis in Leeds was found to be similar to that in the south of the United Kingdom but lower than that in Scotland. There is no evidence of a latitudinal gradient of increasing prevalence of multiple sclerosis from the south to the north of England.
\end{abstract}

(F Neurol Neurosurg Psychiatry 1998;64:605-610)

Keywords: multiple sclerosis; epidemiology; prevalence; latitudinal gradient

Multiple sclerosis is the most common cause of serious neurological disability in young adults in the United Kingdom. It is most prevalent in temperate regions, affecting an estimated 60000 people in the United Kingdom and about two million people world wide. ${ }^{1}$

The first population based study of multiple sclerosis was performed in north Wales in $1929 .^{2}$ Since then numerous prevalence surveys have been carried out disclosing striking differences in the frequency of multiple sclerosis between different parts of the world. In
1950 Limburg first hypothesised that rates of multiple sclerosis rise with increasing distance from the equator. ${ }^{3}$

The prevalence of multiple sclerosis in Orkney, Shetland, and north east Scotland is said to be higher than anywhere else so far studied. ${ }^{4-6}$ Previous studies within the United Kingdom have supported the idea of an increasing latitudinal gradient existing between the south of England and the north of Scotland. ${ }^{7}$ This has been challenged in a study of mortality rates from multiple sclerosis in which the evidence for a north-south gradient was less convincing. ${ }^{8}$ In that study regional mortality rates in England and Wales did not show a clear north-south gradient and there was a reduction in the differential between mortality rates in Scotland and England between the five year periods 1954-8 and 1979-83. Absolute numbers were probably significantly underestimated in this study, however, as only those with multiple sclerosis as the underlying cause of death were considered. Reanalysis of the previous United Kingdom prevalence studies also suggests that the evidence for a gradient in prevalence with latitude is less convincing. ${ }^{9}$

In the past 10 years seven epidemiological studies of multiple sclerosis have been carried out in the southern United Kingdom. ${ }^{10-16}$ In the area between Cambridgeshire (latitudes $52^{\circ}$ to $53^{\circ} \mathrm{N}$ ), and Aberdeen (latitude $57^{\circ}$ to $57.5^{\circ} \mathrm{N}$ ), there has been no recent comparable study of the epidemiology of multiple sclerosis. A prospective study in Rochdale found the prevalence of multiple sclerosis to be similar to that of studies in the southern United Kingdom. ${ }^{17}$ If the evidence for a latitudinal gradient of prevalence in the United Kingdom is being questioned, it is important to use comparable methodology to examine prevalence between these two extremes of latitude. The aim of this study was to determine the prevalence of multiple sclerosis in the north of England, specifically in Leeds Health Authority.

\section{Study area}

Leeds Health Authority serves the local authority area of Leeds with a population estimated to be 732061 as of 1 January 1996 (West Yorkshire Central Services Agency, March 1996). Leeds is situated at a latitude of $53^{\circ} 48^{\prime} \mathrm{C} \mathrm{N}$ and longitude $001^{\circ} 33^{\prime} \mathrm{W}$. It is a predominantly urban district which includes within its boundaries the city, suburbs, and small outlying rural areas. Five clinical neurologists based at two regional centres, St James's University Hospital and the General 
Table 1 Alternative diagnoses of notified patients who did not have multiple sclerosis

\begin{tabular}{ll}
\hline Alternative diagnosis & No \\
\hline Functional disorder & 5 \\
Unrelated medical problems & 4 \\
Cervical myelopathy & 3 \\
Undiagnosed & 3 \\
Peripheral neuropathy & 2 \\
Cerebrovascular disease & 2 \\
Migraine & 2 \\
Polio & 2 \\
\hline
\end{tabular}

Other diagnoses in single cases were: chronic fatigue syndrome, Behçet's disease, Parkinson's disease, Werdnig-Hoffman muscular dystrophy, mononeuritis multiplex, cerebral palsy, motor neuron disease, hereditary spastic paraparesis, white matter disease of unknown aetiology, bilateral simultaneous optic neuropathy, and "squint" ( $\mathrm{n}=11)$

Infirmary at Leeds, are responsible for providing neurology outpatient and inpatient services in the study area and in the surrounding parts of Yorkshire. Further neurological outpatient facilities are provided at two district general hospitals. The study area is served by 409 general practitioners based at 130 practices.

\section{Methods}

SOURCES OF ASCERTAINMENT

Hospital consultants

The diagnostic indices of the three neurologists at St James's University Hospital were examined for all patients seen in whom a diagnosis of multiple sclerosis had been considered or confirmed since 1990. Neurology ward discharges and outpatient attendances were examined at both centres to identify patients discharged with definite, probable, or possible multiple sclerosis since 1990. Patients seen privately were also notified by two neurologists.

\section{Hospital episode statistics}

The computerised listings of hospital inpatient episodes at both centres provided a list of all patients admitted since 1992 with a primary or secondary diagnosis of multiple sclerosis.

\section{General practitioners}

All general practitioners within Leeds Health Authority were asked to provide names, postcodes, and dates of birth of all patients with suspected or confirmed multiple sclerosis. Permission was also sought to allow access to the patients' records if necessary (general practitioners were informed that no patient would be contacted without their prior permission). General practitioners were approached in sequence according to postal district. Three postal reminders were sent to non-responding general practitioners at monthly intervals after

Table 2 Sources of notification of patients with confirmed multiple sclerosis in the Leeds Health Authority

\begin{tabular}{lcc}
\hline Source & No (\% of total) & $\begin{array}{l}\text { Sole source } \\
n(\%)\end{array}$ \\
\hline Neurology departments & $418(59)$ & $141(20)$ \\
General practitioners & $398(56)$ & $82(12)$ \\
Wheelchair Centre & $295(41)$ & $23(3)$ \\
Multiple Sclerosis Society & $140(20)$ & $14(2)$ \\
Multiple Sclerosis Therapy Centre & $151(21)$ & $6(1)$ \\
Community occupational therapists and physiotherapists & $205(29)$ & $6(1)$ \\
Young Disabled Unit & $79(11)$ & $0(0)$ \\
Total & $712(100)$ & - \\
\hline
\end{tabular}

the initial mailing. This was followed by a personal telephone call to the remaining nonresponders.

Multiple Sclerosis Society and the West Yorkshire Multiple Sclerosis Therapy Centre

The chairperson of the Leeds branch of the Multiple Sclerosis Society of Great Britain and Northern Ireland was asked to provide a list of affected members once permission had been obtained from the membership. The West Yorkshire Multiple Sclerosis Therapy Centre was also asked to provide a list of their members.

\section{Community physiotherapists, community} occupational therapists, the Wheelchair Centre and the Younger Disabled Unit

The community physiotherapists, community occupational therapists, and wheelchair centre were asked for a list of their patients with a diagnosis of multiple sclerosis. The attendance records of the Younger Disabled Unit were examined to identify additional patients.

CONFIRMATION OF DIAGNOSIS

The above ascertainment sources were used to form the provisional register. Hospital case notes were then examined to classify patients, according to the Poser criteria. ${ }^{18}$ Clinically definite multiple sclerosis, laboratory supported definite multiple sclerosis, clinically probable multiple sclerosis, and laboratory supported probable multiple sclerosis were collectively categorised as definite and probable multiple sclerosis. These were distinguished from patients with suspected multiple sclerosis and those who did not have multiple sclerosis. The suspected group consisted of those with two episodes of demyelination with no confirmatory clinical signs, those with a single episode of demyelination and clinical evidence of a single lesion only with no paraclinical evidence of a second lesion, and isolated spinal cord syndromes for which no other cause had been identified. A standard proforma was used to classify cases strictly with particular regard to the definition of "attacks" of multiple sclerosis due to the significant between observer variation previously identified in classifying multiple sclerosis using the Poser criteria. ${ }^{19}$ An attack was defined as the occurrence of a symptom or symptoms of neurological dysfunction thought to be due to demyelination, lasting for more than 24 hours with no other identifiable cause. A separate attack was defined as such if it occurred more than one month after a preceding "attack" and clearly involved a distinct area of the CNS. This information had to be documented in the notes for an "attack" to be accepted as such for classification.

Cases were also classified according to the course of the disease with the course recorded as early relapsing-remitting, secondary progressive, relapsing progressive, benign, primary progressive, and unable to classify. This classification was used in a previous study of agreement between observers in classifying multiple sclerosis ${ }^{19}$ and the definitions used 
Table 3 Diagnostic classification of prevalent patients in the Leeds Health Authority

\begin{tabular}{lcc}
\hline Poser classification & No $(\%)$ & Prevalence $\left(10^{5}\right)$ \\
\hline Clinically definite & $320(45)$ & 43.7 \\
$\begin{array}{l}\text { Laboratory supported } \\
\quad \text { definite }\end{array}$ & $63(9)$ & 8.6 \\
Clinically probable & $139(19)$ & 19.0 \\
Laboratory supported & & \\
$\quad$ probable & $4(1)$ & 0.5 \\
Unclassified & $91(13)$ & 12.4 \\
Suspected & $95(13)$ & 13.0 \\
Total & $712(100)$ & 93.7
\end{tabular}

Unclassified $=$ probable or definite multiple sclerosis verified by a consultant neurologist but not classified according to the Poser criteria.

were equivalent to those defined by international consensus with the "relapsing progressive" group of this study identified as "progressive relapsing". ${ }^{20}$ For the definition of course "attacks" were accepted if they involved the same area of the CNS but the reappearance of the symptoms was not the result of other factors such as documented infection, raised temperature, or fatigue. The course was only classified if the patient had been seen by a neurologist in the previous year.

Age at onset was calculated using the date on which the first unequivocal symptom of the disease had occurred. Place of birth, family history, and occupation were also documented.

Patients identified by the Multiple Sclerosis Society or by the Multiple Sclerosis Therapy Centre and not identified by the hospital neurology departments or their general practitioners, were contacted through these organisations and asked to provide details of their general practitioners and their consultant neurologist and permission was sought to access their medical records.

Clinical data were collected by two of us (HF, EG) using a standard proforma to abstract information from clinical records. The cases were classified by two observers with the classification being adjusted if necessary after joint review of the clinical data.

The provisional register was checked with the Family Health Services Authority (FHSA) computer to identify those patients who were resident in the study area, alive and currently registered with a general practitioner. The Leeds FHSA also provided five year age groups and a sex breakdown for all residents of the study area for 1 January 1996. Prevalence was calculated as a point prevalence at the end of the ascertainment period. Thus patients were

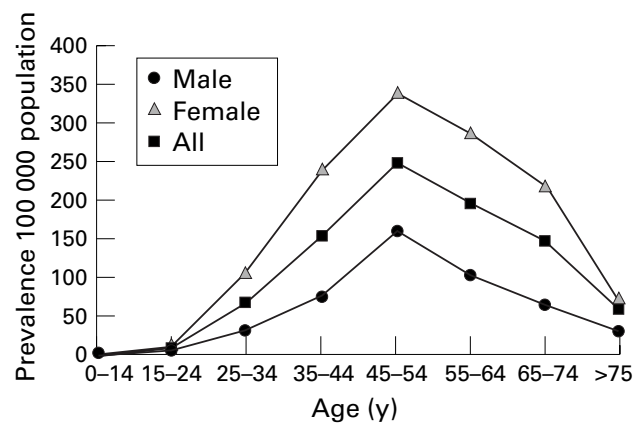

Age and sex specific prevalence of multiple sclerosis in Leeds Health Authority. considered prevalent if they were alive and normally resident in the area on 30 April 1996.

New diagnoses of suspected, probable, or definite multiple sclerosis made by all neurologists in the study area were recorded prospectively from 1 November 1995. All general practitioners were encouraged to maintain the register by informing the study group of any patients with multiple sclerosis joining or leaving their practices, and any newly diagnosed patients in their practices.

\section{STATISTICAL METHODS}

Age and sex specific prevalences were calculated. Standardised prevalence ratios were calculated using the 1961 census population of Northern Ireland as the denominator. ${ }^{21}$

The results were compared with the prevalence surveys in north Cambridgeshire, ${ }^{15}$ south and east Cambridgeshire, ${ }^{13}$ Sussex, ${ }^{16}$ Southampton, ${ }^{12}$ south east Wales, ${ }^{11}$ and Rochdale, ${ }^{17}$ in which the Poser criteria had been used to classify multiple sclerosis.

The study was approved by the local research ethics committees and conformed with the requirements of the Data Protection Act.

\section{Results}

The provisional register contained 1093 people from all sources, $222(20 \%)$ of whom were not resident within the study area.These were excluded from prevalence calculations. Fifty six people died before prevalence day and nine patients were no longer resident in Leeds on the prevalence day, 30 April 1996. Forty six people were identified who did not have multiple sclerosis. Table 1 shows the alternative diagnoses.

Seven hundred and sixty people were identified with multiple sclerosis, alive and prevalent within the study area on 30 April 1996. The largest source of ascertainment of prevalent patients was the hospital neurology departments, which identified 418 (59\%) patients (table 2). Three hundred and seventy one (92\%) general practitioners responded. Twenty seven general practitioners were unwilling to participate due to pressure of work, with the remaining non-responders having retired or left the practices.

The medical records were examined in 712 (94\%) cases. General practice records were examined in 18 cases. Twenty one patients could not be traced on prevalence day, mainly because their date of birth was unknown. In 27 cases the hospital case records were unobtainable. These 48 cases were excluded from further analyses.

The crude prevalence of multiple sclerosis in Leeds on 30 April 1996 was 97.3/10 . Table 3 shows prevalence by diagnostic category. In 91 patients, identified as "multiple sclerosis" the diagnostic assessment of the consultant neurologist was accepted but there was insufficient information to make a classification according to the strict application of the criteria. For "definite and probable" multiple sclerosis including this group the prevalence was $84.5 / 10^{5}$, and for "suspected" cases $13.0 / 10^{5}$. 
Table 4 Comparison of surveys from Leeds, Cambridge, Susssex, Rochdale, Southampton, and south east Wales

\begin{tabular}{|c|c|c|c|c|c|c|c|}
\hline & Leeds 1995 & $\begin{array}{l}\text { Cambridge } \\
1993\end{array}$ & Sussex 1991 & $\begin{array}{l}\text { Cambridge } \\
1990\end{array}$ & $\begin{array}{l}\text { Rochdale } \\
1989\end{array}$ & $\begin{array}{l}\text { Southampton } \\
1987\end{array}$ & $\begin{array}{l}\text { SE Wales } \\
1985\end{array}$ \\
\hline Population & 732061 & 378959 & 596594 & 288410 & 207600 & 417000 & 376718 \\
\hline Cases (n) & 712 & 449 & 665 & 374 & 254 & 411 & 441 \\
\hline Prevalence & 97 & 118 & 136 & 130 & 122 & 99 & 117 \\
\hline Definite or probable & 85 & 107 & 111 & 112 & 96 & 95 & 101 \\
\hline Suspected & 13 & 13 & 24 & 18 & 11 & 4 & 16 \\
\hline Mean age (y) & 51 & 50 & 48.6 & 49.2 & 48.9 & 48.6 & 48.7 \\
\hline Mean age at onset (y) & 34 & 33.8 & 33.1 & 29.6 & 35 & 32.6 & 32.2 \\
\hline Mean duration (y) & 16 & 16.5 & 15.5 & 19.2 & 13.9 & 15.7 & 16.5 \\
\hline Sex ratio F:M & 2.8 & 2.2 & 2.5 & 2.5 & 2.1 & 2.1 & 2.0 \\
\hline SPR & 88 & 139 & - & 121 & 143 & 115 & 139 \\
\hline
\end{tabular}

$\mathrm{SPR}=$ standardised prevalence ratio.

For "definite and probable" classified by the Poser criteria the prevalence was $72.1 / 10^{5}$. The suspected group included 40 patients with isolated spinal cord syndromes, 11 of these having oligoclonal bands in the CSF; 23 patients with two episodes of demyelination involving different areas of the CNS but without documented clinical signs; 24 patients with a single episode of demyelination; and eight patients with recurrent episodes involving the same region but with neither clinical symptoms nor signs of more than one lesion. The standardised prevalence was $88.0 / 10^{5}$ calculated using the 1961 population of Northern Ireland as the reference population. ${ }^{21}$

The figure shows the prevalence of multiple sclerosis in the Leeds Health Authority by age and sex. The mean age of prevalent cases was 51 (SD 13), range 23-86). The sex ratio was 2.79:1 (women:men) with a prevalence in women of $141.0 / 10^{5}$ and in men of $52.0 / 10^{5}$. The mean age at onset of symptoms was 34 (SD 10), range 10-69. The mean duration of disease was 16 (SD 11), range $0-65$ years.

The course of the disease was classified in $481(68 \%)$ cases. Definition of the course was only attempted in those patients who had been seen by a consultant neurologist in the year preceding prevalence day. In some of these cases insufficient information was available in the case notes to accurately define the course of the disease. Of the cases classified according to the course of multiple sclerosis 96 (19\%) were relapsing-remitting, 225 (47\%) secondary progressive, $69(14 \%)$ primary progressive, 38 $(8 \%)$ relapsing progressive, and $53(11 \%)$ benign.

Forty cases were prospectively reported as incident cases between 1 November 1995 and 1 February 1996. All of these cases were ascertained by the neurology departments and all were resident within the study area. This gives a crude estimate of current local incidence of multiple sclerosis of 4.4/100 000/year.

Table 4 is a comparison of the prevalence rates and the standardised prevalence ratios in the studies using the Poser criteria: Leeds, south east Wales, Southampton, south and east Cambridgeshire, north Cambridgeshire, Sussex, and Rochdale. The standardised prevalence is lower than in all of these previous prevalence surveys.

\section{Discussion}

This is the first prevalence study of multiple sclerosis in Leeds and the first major prevalence study of multiple sclerosis in the north of
England since the study of Poskanzer et al of Northumberland and Durham in $1959 .^{22}$ There was a small study of the prevalence of multiple sclerosis in the Calderdale area of west Yorkshire in 1978, in which cases were identified from general practitioners with computerised records in the area. ${ }^{23}$ The prevalence rate recorded was $76 / 10^{5}(n=120)$. A prospective study in Rochdale, Greater Manchester, found a prevalence of $122 / 10^{5}$ in $1989 .{ }^{17}$

The prevalence of multiple sclerosis in the Leeds Health Authority was 97.3/10 . This prevalence is similar to that of Southampton but lower than the other first prevalence surveys using the Poser diagnostic criteria in the south of England and south Wales-south east Wales $1985^{11}\left(117 / 10^{5}\right)$, Southampton $1987^{12}\left(99 / 10^{5}\right)$, south and east Cambridgeshire $1990^{13}\left(130 / 10^{5}\right)$, north Cambridgeshire ${ }^{15}$ $\left(118 / 10^{5}\right)$, and Sussex ${ }^{16}\left(136 / 10^{5}\right)$. There is thus no evidence from this study of a latitudinal gradient of increasing prevalence of multiple sclerosis from the southern United Kingdom to the north of England.

It has been suggested that confidence intervals are an important and often omitted feature of prevalence surveys. ${ }^{9}$ We have not quoted confidence intervals for the prevalence of multiple sclerosis in Leeds. Confidence intervals have relevance only when a sample is considered to have been taken randomly from a population. We do not wish to suggest that the prevalence of multiple sclerosis in Leeds in 1996 applies either to another place or time. A confidence interval would imply that our data were compatible with the "true" prevalence of multiple sclerosis in Leeds being lower than that ascertained.

Fifty nine per cent of the prevalent cases were ascertained by neurology departments and they were the sole source of ascertainment of $20 \%$ of the prevalent population. These cases were apparently not known to their general practitioners. This may be due to the variable quality of record keeping in general practice but it could also reflect neurology services being seen as the main point of medical contact for these people. By contrast, general practitioners were the sole source of ascertainment of $12 \%$ of the prevalent cases. Neurologists' records were examined from 1990 and hospital episode statistics from 1992. People with multiple sclerosis may have had contact with the neurology services before this and not have had continued contact. 
The population studied in this prevalence survey (732 061) was larger than each of the above contemporary studies, and over twice the size of the populations studied in south east Wales and Cambridge. Studies based on very large populations are liable to underascertainment. Studies of small populations are more liable to chance fluctuations. One of the advantages of studying a large population is that local variations are removed, such as the location of a young disabled unit or residential facility for the disabled. In this study 48 patients were excluded from analysis who may subsequently be confirmed to have multiple sclerosis. Unique patients with multiple sclerosis may have been known to the non-participating general practitioners. These factors contribute to underascertainment but in this population would not significantly alter the crude prevalence rate.

To study a large population there are both economic and time constraints. We thus elected to classify patients with multiple sclerosis using case notes only without interviewing or examining patients at home. To minimise diagnostic error we used strict definitions and a standard proforma to abstract information from the case notes. This did not allow us to classify all of the cases in whom the case notes were examined and in $13 \%(n=91)$ of cases the diagnosis of the consultant neurologist was accepted without classification according to the Poser criteria. These criteria were developed not only for use in multicentre therapeutic trials but also to allow comparison between epidemiological surveys. In previous prevalence surveys no definition of "attacks" of multiple sclerosis has been specified and there has been no indication of the level of variation between observers in classification. It is thus difficult to directly compare the Poser groups and it makes the distinction between Poser "definite/probable" and "suspected" disease less clear cut.

In the survey of the Brighton and the Mid-Downs Health Districts, ${ }^{16}$ cases were classified by case notes only. This was said to be to maximise case notifications. In that survey all of the cases were classified. In the surveys of south east Wales ${ }^{11}(36 \%)$, Southampton ${ }^{12}$ $(36 \%)$, south and east Cambridgeshire ${ }^{13}$ (219 $(59 \%))$, and north Cambridgeshire ${ }^{15}(35 \%)$, a varying proportion of cases were classified from case notes alone. However, in the analysis of prevalence by diagnostic category no reference is subsequently made to the source of the diagnostic information. Classification was made in most cases in these surveys into "definite" or "probable" multiple sclerosis and "suspected" multiple sclerosis with only a few cases remaining unclassified. It might be expected that more suspected cases would be diagnosed from case note review alone; however, the prevalence rate for "suspected" cases in this survey is similar to that of south east Wales, ${ }^{11}$ both Cambridge studies ${ }^{13}{ }^{15}$ and Sussex. ${ }^{16}$ The prevalence rate for "suspected" cases in Southampton ${ }^{12}\left(4 / 10^{5}\right)$ was thought to be low due to general practitioners being the main source of ascertainment $(73 \%)$. The large excess in unclassified cases in the present study may be due to rigid application of the criteria. There is no suggestion from previous surveys of lack of diagnostic accuracy in cases classified by notes alone. Although in small populations interview and examination of each case may be optimal, it is important to quantify the gain in diagnostic accuracy as this has important implications for future prevalence studies.

The course of multiple sclerosis was classified in $68 \%$ of cases. Classification of course is difficult from case note review as progression of disease and accumulation of disability are often poorly documented. Most of those patients classified by course were ascertained by neurology departments. This would explain the predominance of progressive disease, $69 \%$ in total (secondary progressive $(47 \%)$, primary progressive $(14 \%)$, and relapsing progressive $(8 \%))$. There has been recent consensus agreement on the definitions of the categories of course of multiple sclerosis. ${ }^{20}$ However, these categories have not been applied to a community based population. In a large population self report of relapses and of progression of disease by postal questionnaire would provide up to date information which may be more accurate than case note review and more cost effective than assessment at home.

The standardised prevalence of $88 / 10^{5}$ is significantly lower than that of each of the studies in table 4 . The age structure of the Leeds population is significantly different from that of the 1961 Northern Ireland survey, in which nearly $30 \%$ of the population was under 15 compared with less than $20 \%$ of the Leeds population. There are many more people in Leeds in the 25-34 year age group and higher proportionally throughout the adult categories. This would explain the significant difference between the crude prevalence and the standardised prevalence. The lower crude and standardised prevalence may also be related to the ethnic group breakdown of the Leeds study population. In the 1991 census (Leeds City Council) $6 \%$ of the people in Leeds described themselves as belonging to an ethnic group other than white, with the predominant groups being Indian (1.5\%), Pakistani (1\%), and Carribean $(1 \%)$. This frequency varies significantly between wards, from $34 \%$ in Chapel Allerton and $30 \%$ in Harehills to $0.5 \%$ in Wetherby and $0.8 \%$ in Otley. There are therefore clearly defined areas with high frequencies of non-white populations. The prevalence of multiple sclerosis in Harehills was 42.3/10 compared with $142.8 / 10^{5}$ in Otley, suggesting a much lower prevalence in an area with high ethnicity. However, the prevalences in Chapel Allerton and Wetherby were similar: $100.1 / 10^{5}$ and $105.5 / 10^{5}$ respectively. Clearly other confounding factors need to be considered including the effect of social class and of ascertainment in inner city areas. In addition there must be caution in drawing conclusions from population figures based on the 1991 census, which was inaccurate, particularly in inner city areas.

The risk of multiple sclerosis is low in Asia, Africa, and the West Indies. ${ }^{24}$ Migration studies have suggested that the risk of multiple 
sclerosis in a single ethnic group varies with place of residence during a critical period of childhood, although the evidence is not straightforward. ${ }^{26}$ The prevalence of multiple sclerosis children born in the United Kingdom of West Indian, African, and Asian immigrants has been claimed to approximate to that seen in similar age groups in the indigenous population. ${ }^{27}$ This study used surnames only for case ascertainment, involved a small number of cases and used a different population, that of Sutton ${ }^{28}$ for comparison. We propose to further study the prevalence of multiple sclerosis in the ethnic groups in the Leeds population to confirm or refute these findings in a well defined population.

HF was funded by the St James's Trust for Nervous System Diseases, and EG was funded by the Nuffield Institute for Health, University of Leeds. We are very grateful to all the people with multiple sclerosis who cooperated with this study.

1 Compston DAS. The dissemination of multiple sclerosis. $\mathcal{f}$ $R$ Coll Physicians Lond 1990;24:207-18.

2 Allison RS Disseminated sclerosis in north Wales. Brain 1931;53:391-430.

3 Limburg CC. The geographic distribution of multiple sclerosis and its estimated prevalence in the United States. Proceedings of the Association for Research into Nervous and Mental Diseases 1950;28:15-24.

4 Poskanzer DC, Prenney LB, Sheridan JL, Yon Kondy J. Multiple sclerosis in the Orkney and Shetland Islands. I: epidemiology, clinical factors, and methodology. $\mathcal{F}$ Epidemiol Community Health 1980;34:229-39.

5 Shepherd DI, Downie AW. A further prevalence study of multiple sclerosis in north-east Scotland. 7 Neurol Neurosurg Psychiatry 1980;43:310-5.

6 Dean G, Goodall J, Downie A. The prevalence of multiple sclerosis in the Outer Hebrides compared with north-east Scotland and the Orkney and Shetland Islands. F Epidemiol Community Health 1981;35:110-3.

7 Swingler RJ, Compston DAS. The distribution of multiple Swingler RJ, Compston DAS. The distribution of multiple
sclerosis in the United Kingdom. I Neurol Neurosurg

8 Williams ES, Jones DR, McKeran RO. Mortality rates from multiple sclerosis: geographical and temporal variations revisited. F Neurol Neurosurg Psychiatry 1991;54:104-9.

9 Robertson N, Compston A. Surveying multiple sclerosis in the United Kingdom. $\mathcal{f}$ Neurol Neurosurg Psychiatry 1995;58:2-6.
10 Williams ES, McKeran RO. Prevalence of multiple sclerosis in a south London borough. BMF 1986;293:237-239.

11 Swingler RJ, Compston DAS. The prevalence of multiple sclerosis in south east Wales. $\mathcal{F}$ Neurol Neurosurg Psychiatry 1988;51:1520-4.

12 Roberts MHW, Martin JP, McLelland L, et al. The prevalence of multiple sclerosis in the Southampton South West Hampshire Health Authority. $\mathcal{F}$ Neurol Neurosurg Psychiatry 1991;54:55-9.

13 Mumford CJ, Fraser MB, Wood NW, et al. Multiple sclerosis in the Cambridge Health District of East Anglia. $\mathcal{F} \mathrm{Neu}$ rol Neurosurg Psychiatry 1992;55:887-92.

14 Sharpe G, Price SE, Last A, et al. Multiple sclerosis in island populations- prevalence in the Bailiwicks of Guernsey and Jersey. $\mathcal{F}$ Neurol Neurosurg Psychiatry 1995;58:22-6.

15 Robertson N, Deans J, Fraser M, et al. Multiple sclerosis in the north Cambridgeshire districts of East Anglia. $\mathcal{F}$ Neurol Neurosurg Psychiatry 1995;59:71-6.

16 Rice-Oxley M, Williams ES, Rees JE. A prevalence survey of multiple sclerosis in Sussex. F Neurol Neurosurg Psychiatry 1995;58:27-30.

17 Shepherd DI, Summers A. Prevalence of multiple sclerosis in Rochdale. F Neurol Neurosurg Psychiatry 1996;61:415-7.

18 Poser CM, Paty DW, Scheinberg L, et al. New diagnostic criteria for multiple sclerosis: guidelines for research protocols. Ann Neurol 1983;13:227-31.

19 Ford HL, Johnson MH, Rigby AS. Variation between observers in classifying multiple sclerosis. $f$ Neurol Neurosurg Psychiatry 1996;61:418.

20 Lublin FD, Reingold SC. Defining the clinical course of multiple sclerosis: results of an international survey. Neurology 1996;46:907-11.

21 Millar JHD. Multiple sclerosis in Northern Ireland. In: Clifford Rose F, ed. Clinical neuroepidemiology. Tunbridge Wells: Pitman, 1980:222-7.

22 Poskanzer DC, Schapira K, Miller H. Epidemiology of multiple sclerosis in the counties of Northumberland and Durham. I Neurol Neurosurg Psychiatry 1963;26:368-76.

23 McCoubrie M, Shuttleworth D. The prevalence of multiple sclerosis in west Yorkshire. BMF 1978;2:570.

24 Martyn C. The epidemiology of multiple sclerosis. In: Matthews WB, Compston A, Allen IV, et al, eds. McAlpines' multiple sclerosis. London: Churchill Livingstone, 1991: $11-8$

25 Dean G, Bhigjee AIG, Bill PLA, et al. Multiple sclerosis in black South Africans and Zimbabweans. F Neurol Neurosurg Psychiatry 1994;57:1064-9.

26 Compston A. Risk factors for multiple sclerosis: race or place? F Neurol Neurosurg Psychiatry 1990;53:821-3.

27 Elian M, Nightingale S, Dean G. Multiple sclerosis among United Kingdom born children of immigrants from the Indian subcontinent, Africa, and the West Indies. 7 Neurol Neurosurg Psychiatry 1990;53:906-11.

28 Williams ES, McKeron RD. Prevalence of multiple sclerosis in a south London Borough. BMF 1986;293:237-9. 